\section{Venter, Craig John}

\author{
A. M. Gressner ${ }^{1}$ und O. A. Gressner ${ }^{2}$ \\ ${ }^{1}$ Labor Dr. Wisplinghoff Berlin, Berlin, Deutschland \\ ${ }^{2}$ Labor Dr. Wisplinghoff Köln, Köln, Deutschland
}

Lebensdaten Amerikanischer Biochemiker, geboren am 14. Oktober 1946 in Salt Lake City, Utah, USA.

Verdienste Craig John Venter publizierte bereits während seiner Promotion über 10 hochrangige wissenschaftliche Arbeiten. Er gründete im Jahr 1998 das Biotechnologieunternehmen Celera Corp., das sich u. a. mit der vollständigen Sequenzierung des menschlichen Genoms beschäftigte. 2001 publizierte Venters Arbeitsgruppe eine vorläufige Gesamtsequenz und Kartierung des humanen Genoms ( $\triangleright$ WholeGenome Sequenzierung (WGS)) und hat damit den internen Wettstreit mit dem seit 1990 laufenden, staatlich finanzierten internationalen Humangenomprojekt (HGP) gewonnen. $\mathrm{Zu}$ diesem Erfolg trug auch die von Venter eingesetzte, sich vom Vorgehen des HGP unterscheidende Strategie der Hochdurchsatzsequenzierung (Shotgun-Sequenzierung) bei. In die von Venter im Jahr 2005 gegründete Firma Synthetic Genomics Inc. brachte er mehrere Tausend Patente ein, um sich u. a. der Nutzung der Ergebnisse in den Biowissenschaften und Medizin zu widmen. Venter gelang es auch, 2007 ein komplettes bakterielles Genom zu synthetisieren. Für diese wissenschaftlichen Leistungen erhielt er zahlreiche Auszeichnungen, u. a. den World Health Award (2002), den PaulEhrlich-und-Ludwig-Darmstädter-Preis (2002) und die National Medal of Science USA (2009).

\section{Literatur}

Venter CJ (2009) Entschlüsselt: Mein Genom, mein Leben. S.Fischer Verlag, Frankfurt am Main 